\title{
Benign tumor finding in temporomandibular joint: Cone Beam CT application and radiographical features of suspected condylar osteochondroma
}

\author{
Indri Kusuma Dewi ${ }^{*}$, Aga Satria Nurrachman² ${ }^{2}$, Lusi Epsilawati ${ }^{3}$ (iD
}

\begin{abstract}
Objectives: This case report is aimed to present a finding of a benign tumor at the Temporomandibular Joint (TMJ) area involving the condylar head of the mandible that radiographically showed the typical features of osteochondroma using and emphasizing on the application of Cone Beam CT (CBCT) imaging.
\end{abstract}

Case Report: A 24-year-old female patient came to the Radiology Department of Unpad Dental Hospital as referred from her previous dental surgeon to get $\mathrm{CBCT}$ examination of her entire right side of mandible with a provisional diagnosis of mandibular hyperplasia.

Conclusion: Osteochondroma and condylar hyperplasia are often clinically difficult to differentiate, CBCT imaging can easily distinguish the enlargement of condylar head in condylar hyperplasia with irregular condylar mass and altered trabecular pattern in osteochondroma. CBCT may be helpful in establishing the diagnosis of condylar tumors originating from bone.
${ }^{1}$ Dentomaxillofacial Radiology Residency Program, Faculty of Dentistry Universitas Padjadjaran, Bandung, Indonesia, 40132

${ }^{2}$ Department of Dentomaxillofacia Radiology, Faculty of Dental Medicine, Universitas Airlangga, Surabaya, Indonesia 60132

${ }^{3}$ Department of Dentomaxillofacial Radiology, Faculty of Dentistry, Universitas Padjadjaran, Bandung, Indonesia, 40132

Correspondence to:

Indri Kusuma Dewi

凶indri19003@mail.unpad.ac.id

Received on: February 2021

Revised on: March 2021

Accepted on: April 2021

Keywords: Benign tumor, temporomandibular joint, cone beam CT, osteochondroma

Cite this article: Dewi IK, Nurrachman AS, Epsilawati L. Benign tumor finding in temporomandibular joint: Cone Beam CT application and radiographical features of suspected condylar osteochondroma. Jurnal Radiologi Dentomaksilofasial Indonesia 2021;5(1)23-6. https://doi.org/10.32793/jrdi.v5i1.677

\section{INTRODUCTION}

Benign tumors involving or originating in the Temporomandibular Joint (TMJ) typically manifests as an irregularly shaped enlargement of the condylar head. There may also be diminished trabecular density due to bony destruction or elevated density due to new, abnormal bone formed by the tumor. ${ }^{1}$ Some benign lesions including condylar hyperplasia, osteoma, osteoblastoma, osteochondroma, chondroma chondroblastoma, fibro-osseous lesions, giant cell tumor and vascular malformations were reported to occur in the condylar area. ${ }^{2}$

This case report is aimed to present a finding of a benign tumor at the Temporomandibular Joint (TMJ) area involving the condylar head of the mandible that radiographically showed the typica features of osteochondroma using and emphasizing on the application of Cone Beam CT (CBCT) imaging.

\section{CASE REPORT}

A 24-year-old female patient came to the Department of Dentomaxillofacial Radiology of Unpad Dental Hospital as prescribed from her previous oral surgeon to get CBCT examination for detailed evaluation of her entire right side of mandible with a provisional diagnosis of mandibular hyperplasia. Clinical extra-oral examination (Figure
1) showed progressive facial asymmetry and limitation in mouth opening with some deviation of midline to the left side. No tangible swelling was noticed in both preauricular region.

The CBCT radiography was performed, showing a solitary corticated and irregularly-shaped, roughly oval, mixed radiopaque and radiolucent structure of enlargement, containing trabecular pattern, with diameter of $8-10 \mathrm{~cm}$, pedunculated and adhered on the superior surface of the right condylar head. The borders appeared well-defined and the join the internal structure was continuous with the condylar head. No periosteal reaction was seen around.

The coronal view of CBCT showed the area of lesion is about $226,36 \mathrm{~mm}^{2}$ in size with density (ROI) of 523,4 gray values (Figure 3A). It also clearly revealed that the lesion is continuous and adhered with the condylar head (Figure $3 \mathrm{~B}$ and $4 \mathrm{~A}$ ) on its posterosuperior lateral surface and showed condylar asymmetry (Figure 2, 3A and 5C). CBCT revealed that the lesion extends from the posterior into the superoanterior aspects of the condyle with the mediolateral width of the base is around 9,8 $\mathrm{mm}$ (Figure 4). Based on patient's history, clinical presentation and radiological findings, early differential diagnosis including osteochondroma, osteoma, chondroma, chondroblastoma and other similar bony lesions were included. 


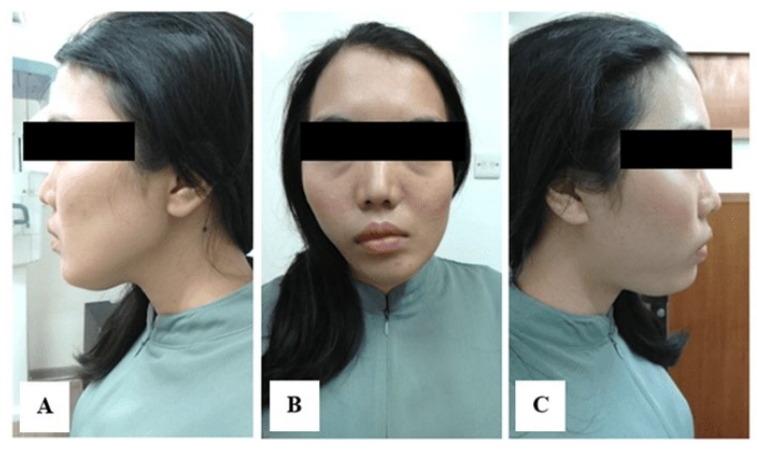

Figure 1. Clinical examination of the patient showed facial asymmetry; A. Left view, B. Front view, C. Right view

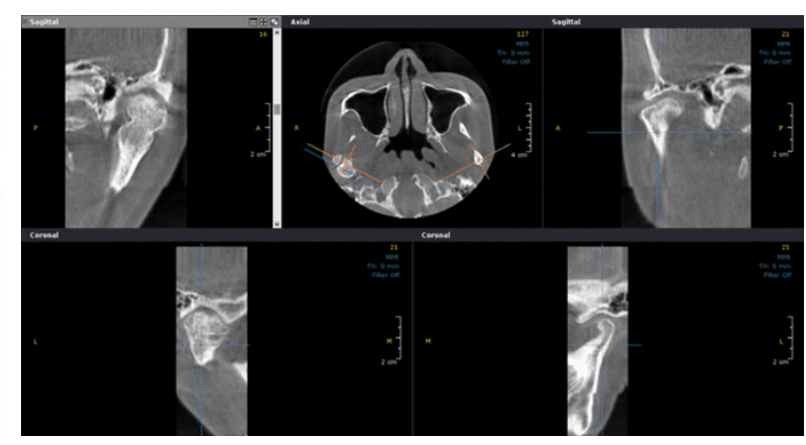

Figure 2. Bilateral TMJ view of CBCT examination

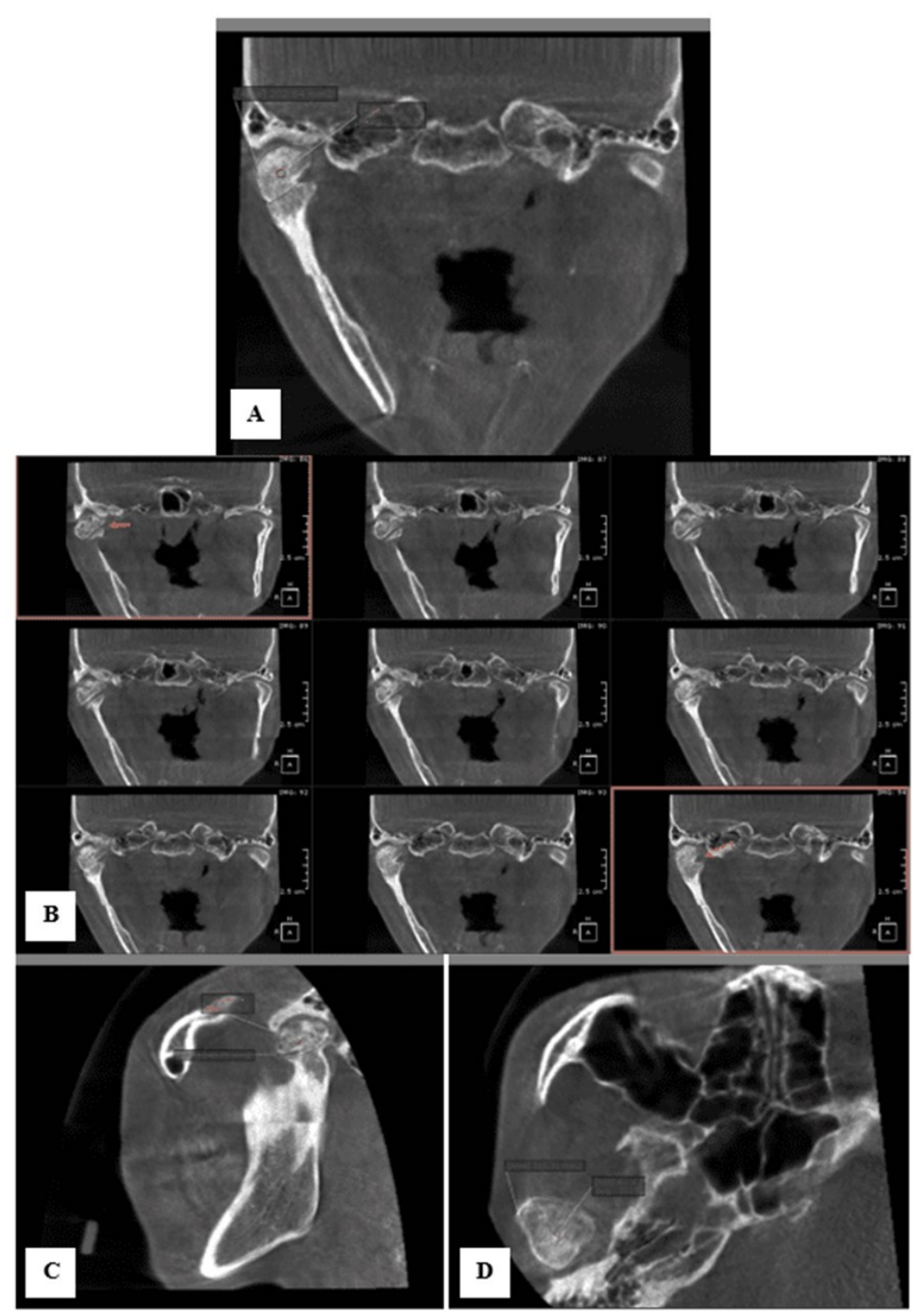

Figure 3. Full $\mathrm{CBCT}$ examination of the right condylar area; A. Single coronal view, B. Slices coronal view, C. Sagital view, D. Axial view

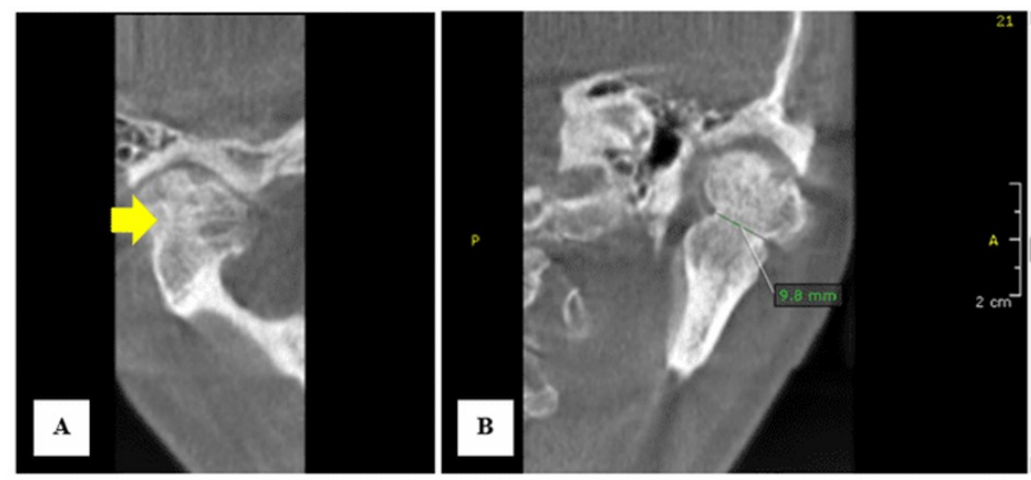

Figure 4. Specific view of the right condyle; A. Coronal, B. Sagittal 

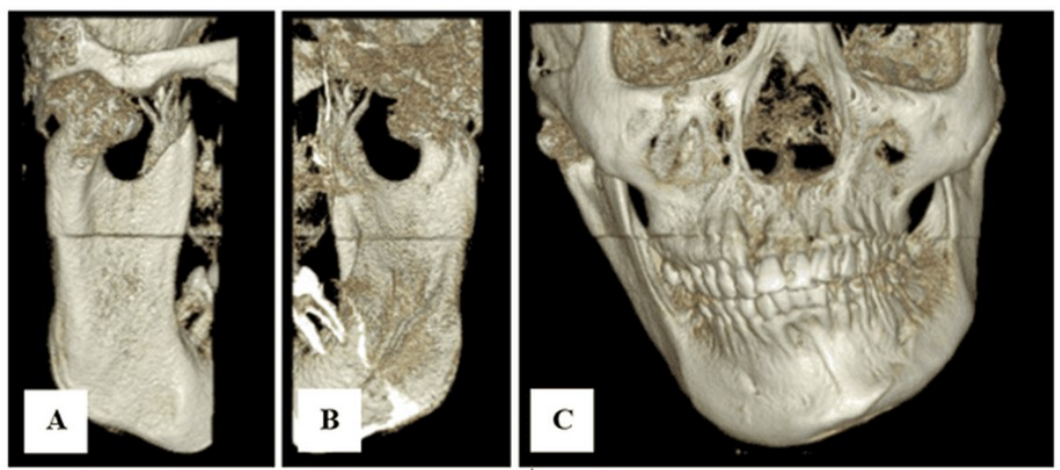

Figure 5. 3D view of CBCT examination; A. Lateral view, B. Medial view, C. Frontal view

\section{DISCUSSION}

Primary neoplasms of the TMJ are incredibly uncommon. The TMJ is composed of hard and soft tissues such as the temporal bone, condyle, articular disc, capsule, and ligaments, and tumors in this area may arise from both bone and soft tissue. ${ }^{2}$ Advanced imaging modalities should be used when there is a suspect of a tumor around this area as conventional methods will not give adequate results to point out the possible differential diagnosis. CBCT is one of advanced modalities in dentistry that also has been used widely for TMJ cases. Although it is not considered as the best tool to evaluate TMJ due to its complex anatomy, the results of a CBCT scan could be necessary to indicate calcified cartilage or delineate soft-tissue changes caused by a tumor development. ${ }^{3}$

All tumors and tumor-like conditions affecting the TMJ exhibit similar clinical presentations. Patients may complain of swelling, pain and reduced range of motion, which frequently mimic TMJ dysfunction. It may also show a facial asymmetry, malocclusion, and deviation of the mandible to the unaffected side. ${ }^{1}$ Benign tumors differ radiographically from malignancies in the TMJ as malignant lesions manifest with a variable degree of bone destruction, with more ill-defined, non-corticated and irregular borders. Most malignant neoplasms also show the distinct area of bone resorption or destruction due to lack of new bone formation with minimal expansion and erosive lesion, which did not appear in this case. ${ }^{4}$ Benign tumors develop slowly in contrast and may reach a certain size before turning into clinically noticeable. $^{1}$

The $\mathrm{CBCT}$ radiograph showed an irregularlyshaped mixed radiopaque and radiolucent structure of enlargement adhered on the posterosuperior lateral surface of the right condylar head. The provisional diagnosis of hyperplasia was neglected as the imaging characteristic of the lesion did not in line in which condylar shape and proportions are better preserved in case of condylar hyperplasia. The enlargement of condylar head in condylar hyperplasia with irregular condylar mass and altered trabecular pattern in osteochondroma clearly differ between the two. ${ }^{1}$

Benign neoplasms, such as osteoma osteoblastoma, chondroma, chondroblastoma and osteochondroma are known to commonly develop in condylar area. ${ }^{2}$ The differential diagnosis of osteochondroma, osteoma, chondroma and chondroblastoma were thus included in the present case based on the clinical and imaging features.

The mixed internal structure of the lesion and the continued cortical border from the parent bone structures suggests the typical features of TMJspecific benign tumors originating from condylar bone, with the main suspect of condylar cartilage tumor, suggestively more considered as an osteochondroma. Osteoma may be ruled out because it normally has a narrow base and also does not show trabecular continuity as it arises from the periosteal surface, as opposed to the wide base and internal structure seen in our case. ${ }^{1,5}$ Chondroma is characterized by the presence of a more widely radiolucent and irregular mass, or well -defined lobules, while chondroblastoma usually manifests as a well-defined lytic geographic lesion with resorptive defects or as condylar enlargements with cortex thinning. ${ }^{2,5}$ Because of the rarity of this tumor, imaging-based criteria for diagnosing chondroblastoma of the TMJ have not been clearly established. ${ }^{6}$ Although it appears as circular radiolucent lesions with sharp boundaries and a thin sclerotic margin in the extremities, the lesion in the TMJ could not be detected as the same in CBCT, and $\mathrm{MRI}$ is considered a more sensitive imaging modality for this case. Chondroblastoma may cause the destruction of the squamous portion of the temporal bone, the glenoid fossa, and the zygomatic arch. ${ }^{2,6,7}$

Osteochondromas is one of the most common benign bone tumors involving the TMJ, accounting for $20-50 \%$ of benign bone tumors and $10-15 \%$ of primary bone tumors, that usually appear as a bony growth, having the appearance of being attached to, or growing from, the condyle., Osteochondromas generally have a more irregular appearance and show a characteristic feature of cartilage-capped bony projection consists of a marrow cavity with the cortex and medulla of the lesion being continuous with the underlying internal cancellous bone of the mandibular condyle. The etiopathology of osteochondroma have not been well understood, but traumatic, developmental, neoplastic and reparative circumstances were reported as possible factors. ${ }^{2}$ Diagnosis is usually made before 30 years of age and may occur at any 
site of the mandibular condyle, with predilection extending in the anterior and medial aspects of the condyle. ${ }^{9,10}$ However, the lesion was found on the posterior and lateral surface of the condyle in this patient. It is incredibly rare for osteochondroma to be involved on both sides of condylar process. ${ }^{11}$ Recurrent osteochondroma was also reported in a few patient after treated. ${ }^{5}$ Other clinical characteristics such as onset at an older age, rapid growth, and invasion to surrounding structures can indicate a possible complication of malignant shifting. ${ }^{12}$

Osteochondroma is a bone disorder that can also be detected using CBCT. Conventional radiography, such as panoramic images, are limited to just two dimensions (2D), causing anatomical superimposition. $\mathrm{CBCT}$ overcomes this limitation as it allows physicians to evaluate complex cases in the maxillofacial area and provides information that aids in the more precise and detailed diagnosis of certain TMJ pathological conditions. ${ }^{13}$ In this case of suspected condylar osteochondroma, CBCT was used as an alternative to CT or MRI. Even though the imaging characteristics were identical, $\mathrm{CBCT}$ may display a more clearly trabecular structure and the base between the tumor and normal bone than CT. ${ }^{10,14}$

Based on CBCT analysis, the lesion was classified as either bony outgrowth or condylar expansion. The first type was discovered in variety of forms such as tongue-like, comb-like, mushroom-like, oval and irregular. Most bony neoplasms did not have a round margin, but rather a wedge angle, thus it is crucial when distinguishing osteochondroma from other pathologic lesions. Since it is histologically formed by cartilage components and calcification, the radiographic presentation of osteochondroma normally shows mixed density on CBCT images; the greater the ossification of the cartilage components, the higher the tumor density. ${ }^{10}$

No further observation and follow-up whether histopathological or another advanced imaging examination is done for the patient to get the conclusive diagnosis. The biopsy itself may be difficult to conduct due to its anatomical complexity and the risk of facial nerve injury. ${ }^{15}$ A confirmatory diagnosis may be obtained only through surgical exploration. In this case, we as the radiologist delivered the imaging interpretation and expertise opinion for the patient and the referral dentist as it is up to their decision for the next treatment.

\section{CONCLUSION}

Cone Beam CT in TMJ may be helpful in establishing the diagnosis of condylar tumors originating from bone, including cartilage tumors such as osteochondroma. Osteochondroma and condylar hyperplasia are often clinically difficult to differentiate, CBCT imaging can easily differentiate between the two. However, it should be emphasized that CBCT diagnostic capability is still limited which is not considered to evaluate soft tissue abnormalities in TMJ.

\section{ACKNOWLEDGMENTS}

None.

\section{FOOTNOTES}

All authors have no potential conflict of interest to declare for this article. Informed consent was obtained from the patient for being included in this case report.

\section{REFERENCES}

1. Mallya SM, Lam EWN. White and Pharoah's Oral Radiology: Principle and Interpretation. 8th Ed. Toronto, Canada: Elsevier; 2018.

2. Yurttutan ME, Öncül AT, Karasu HA. Benign Tumors of Temporomandibular Joint. Temporomandibular Jt Pathol Curr Approaches Underst. 2018;

3. Bachani L, Lingappa A, Shankaramurthy S, Parameshwarappa S. Osteochondroma involving mandibular condyle. J Indian Acad Oral Med Radiol. 2017;29(1):56.

4. Kamdar R, Subbarao C, Ganapathy D. Role of cone beam computed tomography in dentistry. Drug Invent Today. 2019;11(Special Issue 2):145-8.

5. Kwon YE, Choi KS, An CH, Choi SY, Lee JS, An SY. Recurrent osteochondroma of the mandibular condyle: A case report. Imaging Sci Dent. 2017;47(1):57-62.

6. Marano R, Dias do Nascimento Neto C, Mayrink G, Tajra R, Gaigher E. A rare case of chondroblastoma of the temporomandibular joint: A case report. Oral Maxillofac Surg Cases [Internet]. 2019;5(3):100102.

7. Mahammad D, Chingiz R, Elchin A, Farinaz I, Vugar Q. Chondroblastoma of the TMJ: Case Report. Balk J Dent Med [Internet]. 2017 Nov 27;21(3):176-8.

8. Garrison RC, Unni KK, McLeod RA, Pritchard DJ, Dahlin DC Chondrosarcoma arising in osteochondroma. Cance [Internet]. 1982 May 1;49(9):1890-7.

9. Afonso PD, Isaac A, Villagrán JM. Chondroid Tumors a Incidental Findings and Differential Diagnosis between Enchondromas and Low-grade Chondrosarcomas. Semin Musculoskelet Radiol. 2019;23(1):3-18.

10. Liu $Y$, Xiao $Y$, Wang $H, H u D$, Han $X$. Clinical and radiological analysis of osteochondromas of the mandible using conebeam computed tomography. Oral Radiol. 2017;33(1):8-15.

11. Kamble V, Rawat J, Kulkarni A, Pajnigara N, Dhok A Osteochondroma of bilateral mandibular condyle with review of literature. J Clin Diagnostic Res. 2016;10(8):TDO1-2.

12. Harish $M$, Manjunatha BS, Kumar AN, Alavi YA Osteochondroma $(\mathrm{OC})$ of the condyle of left mandible: A rare case. J Clin Diagnostic Res. 2015;9(2):ZD15-6.

13. Tantanapornkul W, Dhanuthai K, Sinpitaksakul P, Itthichaisri $C$ Kamolratanakul $P$, Changsirivatanathamrong V. Dentofacial Deformity Caused by Bulky Osteochondroma: Report of an Unusual Case and the Importance of Cone Beam Computed Tomography. Open Dent J. 2017;11(1):237-41.

14. Larheim TA, Abrahamsson A-K, Kristensen M, Arvidsson LZ. Temporomandibular joint diagnostics using cone beam computed tomography. Dentomaxillofac Radiol [Internet]. 2014; 20140235 .

15. Meshram V Natarajan $C$, Landge J, Jadhav S. Expansile radiolucent lesion of the Temporomandibular Joint-A diagnostic enigma. J Oral Biol Craniofacial Res [Internet]. 2018;8(3):203-5. 